\title{
TIME-DOMAIN HARMONIC STATE ESTIMATION OF NONLINEAR LOAD POWER SYSTEMS WITH UNDER-DETERMINED CONDITION BASED ON THE EXTENDED KALMAN FILTER
}

\author{
Rafael Cisneros-Magaña ${ }^{1,{ }^{*}}$, Aurelio Medina ${ }^{1}$ and Olimpo Anaya-Lara ${ }^{2}$ \\ ${ }^{1}$ Facultad de Ingeniería Eléctrica, División de Estudios de Posgrado, Universidad Michoacana de San \\ Nicolás de Hidalgo, Morelia, México. \\ ${ }^{2}$ Institute for Energy and Environment, Department of Electronic and Electrical Engineering, University \\ of Strathclyde, Glasgow, U.K. \\ * Corresponding author: Facultad de Ingeniería Eléctrica, División de Estudios de Posgrado, Universidad \\ Michoacana de San Nicolás de Hidalgo, Ciudad Universitaria, C.P. 58030, Morelia, Michoacán, México, \\ Tel/Fax: + 52 (443) 327-97-28. \\ e-mail addresses: rcisneros@dep.fie.umich.mx (R. Cisneros-Magaña), amedinr@gmail.com (A. Medina), \\ olimpo.anaya-lara@strath.ac.uk (O. Anaya-Lara).
}

\section{SUMMARY}

This contribution presents a time-domain methodology for harmonic state estimation of power systems with nonlinear loads based on the extended Kalman filter (EKF). The output variables measurements to be used in the state estimation algorithm are selected from the simulation of the propagated harmonics in the system with an under-determined condition of the measurement matrix. The state estimation results are compared against the actual time-domain system response; both results closely agree hence verifying the effectiveness of the EKF to solve the time-domain power system state estimation. Several sampling frequencies and measurement noise are applied to assess the effects on the state estimation process, the error covariance matrix, residuals and on the execution time.

Keywords: Extended Kalman Filter; harmonics; noise; nonlinear network; sampling frequency; state estimation.

\section{INTRODUCTION}

Power Quality State Estimation (PQSE) is particularly relevant to assess the power system dynamic operation through any of its main variants, i.e. Harmonic State Estimation (HSE) [1], Transient State Estimation (TSE) [2] and Voltage Sag Estimation (VSSE) [3-5]. The HSE has been treated mainly in the frequency-domain with different important contributions publicly available [1], [6-7]. The frequencydomain HSE methodology requires the solution for each harmonic under analysis. The principle of superposition is used to obtain the total harmonic distortion throughout the power system. A novelty on 
this paper is that the HSE is solved in the time-domain through the extended Kalman filter (EKF) [8-10] applied to power systems with time-varying nonlinear components with an under-determined system condition. This allows defining fewer measurements than states making the analysis and the state estimation more convenient in the time-domain as highly distorted waveforms may appear. The timedomain harmonic state estimation implicitly takes into account a wide, not limited, range of harmonics of the voltage and current waveforms. Besides, the EKF technique can be applied in time domain representation of power systems with time-varying nonlinear models.

The main objective of HSE is to estimate the harmonic levels in the power network using a mathematical model and a limited number of noisy measured data [1], [6]. The model and its parameters, the choice of measurement points and quantities to be measured, are important concerns to be taken into account. Initially, the analogue signals are sampled and then analog/digital converted. The sampling frequency is related with the number of points per cycle in the discrete time solution and the measurements have to be synchronized; this HSE requirement is solved with the measurement time stamping [1]. A determined number of cycles defined in the measurement window are monitored and the synchronized measurements are sent to a control center in order to be concentrated and processed to solve the state estimation. The discrete waveforms of a partial number of voltages, currents and other power system variables, such as real or reactive power in a time interval or measurement window are the input data to the HSE. This solution is based on a time sequence of synchronized measurements and on the power system model [1], [8], [11].

The HSE follows variations in the waveforms including harmonics and transients by means of the EKF approach, by using the criterion of minimizing the error between the measured and estimated values [12-13]; this residual indicates the accuracy of the estimation and dynamically reflects the fluctuations of the state variables [6]. In the time-domain, the power system model can be represented by a set of nonlinear differential equations. The time varying nonlinear components in the power system originate the injection of harmonics and consequently voltage and current waveform distortion [14-15]. The HSE takes a limited number of measurements and globally estimates the voltage and current harmonics at nodes and elements of the system where they are not monitored [16].

The main motivation to propose a methodology based on EKF is the solution of HSE when the power network is more accurately represented by nonlinear models to include time-varying loads, such as electric arc furnaces and thyristor controlled devices, among others, or to include nonlinear modelling for synchronous generators, as in [17-18]. This nonlinear state estimation can be solved using the EKF. 
This paper proposes the EKF methodology to assess the HSE in Section 2; Section 3 presents the case studies to verify the proposed formulation, and Section 4 draws the main conclusions of the reported research work. Appendices give the test power system parameters, nonlinear electrical load models, definition of state transition matrix $\boldsymbol{\Phi}$ and of measurements matrix $\boldsymbol{H}$.

\section{METHODOLOGY}

The proposed EKF methodology to solve the HSE consists of the following four steps:

a) The measurement period is initiated using a radio or a GPS signal. This signal is sent simultaneously to all monitoring sites equipped with remote terminal units (RTU); voltage and current waveforms are synchronously monitored and recorded during several cycles at predetermined locations of the power system.

b) The measurements set is collected from the power system monitored sites to a control center through suitable communication links.

c) After receiving the measurement set, the EKF is numerically applied using the power system model and the measurement equation to assess the HSE; the estimated state vector is the result of this step.

d) The global system state is obtained using the estimated state and the power system configuration.

This method can be repetitively applied to assess different periods of time.

The power system model considering the time variant or non-autonomous case by means of a set of nonlinear differential equations, is,

$$
\begin{aligned}
& d \boldsymbol{x} / d t=\boldsymbol{f}(\boldsymbol{x}, \boldsymbol{u}, \boldsymbol{v}) \\
& \boldsymbol{y}=\boldsymbol{g}(\boldsymbol{x}, \boldsymbol{u}, \boldsymbol{w})
\end{aligned}
$$

where $\boldsymbol{x}$ is the state vector, $\boldsymbol{f}$ the system function, $\boldsymbol{u}$ the input vector, $\boldsymbol{v}$ the process noise, $\boldsymbol{y}$ the known output vector, $\boldsymbol{g}$ the output system function, and $\boldsymbol{w}$ the measurement noise vector; matrices and vectors are represented in boldface type. An iterative process can be defined for the discrete time nonlinear case, through the conversion of the model from continuous to discrete time. By approximating the differential equations to difference equations [8], when the time step is sufficiently small, the approximate time derivative of $\boldsymbol{x}$ is,

$$
d \boldsymbol{x} / d t=\left(\boldsymbol{x}_{k}-\boldsymbol{x}_{k-1}\right) / \Delta t
$$

Solving for $\boldsymbol{x}_{k}$ and substituting the derivative with (1) yields,

$$
\boldsymbol{x}_{k}=\boldsymbol{x}_{k-1}+\Delta t \boldsymbol{f}\left(\boldsymbol{x}_{k-1}, \boldsymbol{u}_{k-1}, \boldsymbol{v}_{k-1}\right)
$$


Equation (4) gives the discrete nonlinear system model. The discrete expression for the measurement or observation equation is,

$$
\boldsymbol{z}_{k}=\boldsymbol{h}\left(\boldsymbol{x}_{k}, \boldsymbol{u}_{k}, \boldsymbol{w}_{k}\right)
$$

The measurement vector $\boldsymbol{z}$ is defined with the selected output variables to be monitored. These variables are part of the output vector $\boldsymbol{y}$ defined in (2). The process noise $\boldsymbol{v}$ and the measurement noise $\boldsymbol{w}$ are assumed stationary, zero mean and with no correlation among them [1], i.e.

$$
\begin{aligned}
& E\left[\boldsymbol{v}_{k}\right]=0 \\
& E\left[\boldsymbol{v}_{k} \boldsymbol{v}_{k}^{T}\right]=\boldsymbol{Q}_{k} \\
& \boldsymbol{v}_{k} \approx \mathrm{N}\left(0, \boldsymbol{Q}_{k}\right) \\
& E\left[\boldsymbol{w}_{k}\right]=0 \\
& E\left[\boldsymbol{w}_{k} \boldsymbol{w}_{k}^{T}\right]=\boldsymbol{R}_{k} \\
& \boldsymbol{w}_{k} \approx \mathrm{N}\left(0, \boldsymbol{R}_{k}\right)
\end{aligned}
$$

$\boldsymbol{Q} n \times n$ and $\boldsymbol{R} m \times m$ are the process and measurement noise covariance matrices associated with $\boldsymbol{v}$ and $\boldsymbol{w}$ noises, respectively, $n$ states and $m$ measurements; if these noises are uncorrelated, $\boldsymbol{Q}$ and $\boldsymbol{R}$, are diagonal matrices. Figure 1 illustrates the methodology to obtain the time-domain HSE solution of the power system using the EKF.

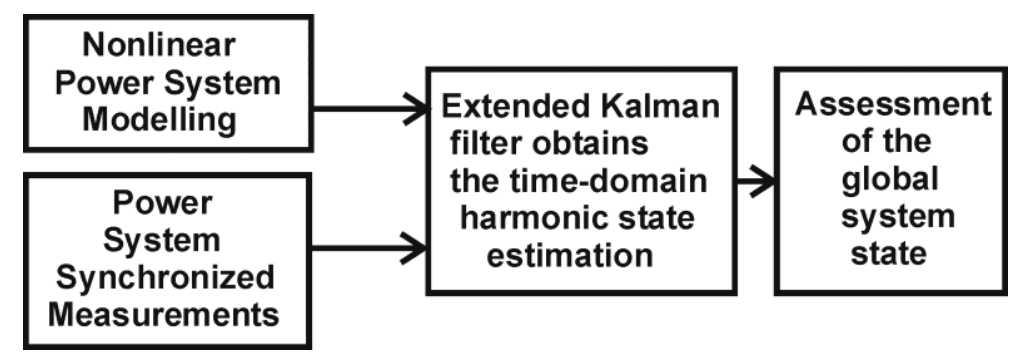

Figure 1. Harmonic state estimation based on the Extended Kalman Filter.

The criterion of validation for the proposed methodology is defined by the normalized root mean square error (NRMSE). This error is frequently used to measure the differences between predicted values by a state estimator and the values actually observed; lower values indicate less estimation residuals. The NRMSE is given by, 


$$
N R M S E=\frac{\sqrt{\frac{\sum_{t=1}^{n p}\left(\hat{\boldsymbol{y}}_{t}-\boldsymbol{y}\right)^{2}}{n p}}}{\boldsymbol{y}_{\max }-\boldsymbol{y}_{\text {min }}}
$$

Where the estimated vector is $\hat{\boldsymbol{y}}$, the observed vector is $\boldsymbol{y}$, and $n p$ the number of data.

\subsection{Extended Kalman Filter}

The EKF takes the model (4) and the measurement model (5), to effectively track the system dynamics in the time-domain and to evaluate the state and output variables during the study interval, taking into account partial measurements from the system [19-22]. Sources, harmonic levels and transients in a power system vary with time and can be followed with the EKF algorithm.

The EKF is based on a Taylor series approximation; its main steps are initialization, time update or prediction and measurement update or correction; time and measurement updates are evaluated at each time step [8], [23-26]. The monitored variable waveforms defined in the measurements vector $z$ are sampled from the power system in a discrete form. With these data, the recursive EKF can be applied. It consists of the following steps:

a) Initial evaluation:

$$
\begin{aligned}
& \hat{\boldsymbol{x}}_{0}^{+}=E\left[\boldsymbol{x}_{0}\right] \\
& \boldsymbol{P}_{0}^{+}=E\left[\left(\boldsymbol{x}_{0}-\hat{\boldsymbol{x}}_{0}^{+}\right)\left(\boldsymbol{x}_{0}-\hat{\boldsymbol{x}}_{0}^{+}\right)^{T}\right]
\end{aligned}
$$

$E$ is the expected value, $\boldsymbol{P}$ is the error covariance matrix, + indicates a posteriori or after measurement estimate and - a priori or before measurement estimate. The subscripts $k$ and $k-1$ denote time instants $t=k \Delta t$ and $t=(k-1) \Delta t$, respectively.

b) Time update or prediction [8], [21]:

b.1) Partial derivative matrix determination,

$$
\boldsymbol{\Phi}_{k-1}=\partial \boldsymbol{f}_{k-1} /\left.\partial \boldsymbol{x}\right|_{\hat{\boldsymbol{x}}_{k-1}^{+}}
$$

The state transition matrix $\boldsymbol{\Phi}$ is of $n \times n$ order.

b.2) Time update or prediction of the state estimate and the error covariance matrix,

$$
\hat{\boldsymbol{x}}_{k}^{-}=\boldsymbol{f}_{k-1}\left(\hat{\boldsymbol{x}}_{k-1}^{+}, \boldsymbol{u}_{k-1}, \boldsymbol{v}_{k-1}\right)
$$




$$
\boldsymbol{P}_{k}^{-}=\boldsymbol{\Phi}_{k-1} \boldsymbol{P}_{k-1}^{+} \boldsymbol{\Phi}_{k-1}^{T}+\boldsymbol{Q}_{k-1}
$$

c) Measurement update evaluation:

c.1) Partial derivative matrix determination,

$$
\boldsymbol{H}_{k}=\partial \boldsymbol{h}_{k} /\left.\partial \boldsymbol{x}\right|_{\hat{\boldsymbol{x}}_{k}^{-}}
$$

The measurements matrix $\boldsymbol{H}$ is of $m \times n$ order.

c.2) EKF gain assessment, update the state estimate and error covariance matrix are calculated as,

$$
\begin{aligned}
& \boldsymbol{K}_{k}=\boldsymbol{P}_{k}^{-} \boldsymbol{H}_{k}^{T}\left(\boldsymbol{H}_{k} \boldsymbol{P}_{k}^{-} \boldsymbol{H}_{k}^{T}+\boldsymbol{R}_{k}\right)^{-1} \\
& \hat{\boldsymbol{x}}_{k}^{+}=\hat{\boldsymbol{x}}_{k}^{-}+\boldsymbol{K}_{k}\left[\boldsymbol{z}_{k}-\boldsymbol{h}_{k}\left(\hat{\boldsymbol{x}}_{k}^{-}, \boldsymbol{u}_{k}, \boldsymbol{w}_{k}\right)\right] \\
& \boldsymbol{P}_{k}^{+}=\left(\mathbf{I}-\boldsymbol{K}_{k} \boldsymbol{H}_{k}\right) \boldsymbol{P}_{k}^{-}
\end{aligned}
$$

The EKF gain $\boldsymbol{K}$ evaluates the estimate of the state variables and neglects the influence of noise in inputs [24]; this gain is a time-varying quantity (19). $\boldsymbol{P}$ is the error covariance matrix, (21) represents the dynamics of $\boldsymbol{P}$, in the sense that there is a recursive relationship between (17) and (21). Equation (20) updates the state estimation with the projected state (16) by multiplying the EKF gain with the residual of the actual measurement vector $\boldsymbol{z}_{k}$ and the estimated measurements.

In this research work, the EKF is applied to estimate the harmonics in a power system with nonlinear loads and with an under-determined condition in the measurement equation (5), that is, less measurements than states. The state estimate $\hat{\boldsymbol{x}}$ and the error covariance matrix $\boldsymbol{P}$ are evaluated recursively in each time step. References [25-29] report different case studies using the Kalman Filter for linear systems; in [17] state and input estimation using the EKF is reported.

The Discrete Fourier Transform (DFT) is used to process the measured and estimated signals to extract their harmonic components, i.e. to transform time-domain data into frequency-domain [30-31].

\subsection{Nonlinear least squares and singular value decomposition.}

The nonlinear least squares (NLS) solves the state estimation if the system is over $(m>n)$, or normaldetermined $(m=n)$. The nonlinear least squares estimation attempts to minimize the square of errors between a known set of measurements and a set of weighted nonlinear functions [32], i.e.

$$
\text { minimize }\|\boldsymbol{e}\|^{2}=\boldsymbol{e}^{T} \boldsymbol{e}=\sum_{i=1}^{m} \frac{1}{\sigma_{i}^{2}}\left[\boldsymbol{z}_{i}-\boldsymbol{h}_{i}(\boldsymbol{x}, \boldsymbol{u}, \boldsymbol{w})\right]^{2}
$$


Where $\boldsymbol{x}$ is the state vector to be estimated, $\boldsymbol{z}$ is the vector of measurements, $\sigma_{i}^{2}$ is the variance of the $i$-th measurement, and $\boldsymbol{h}(\boldsymbol{x}, \boldsymbol{u}, \boldsymbol{w})$ is the nonlinear function vector relating $\boldsymbol{z}$ to $\boldsymbol{x}$; as defined $\boldsymbol{u}$ is the input vector and $\boldsymbol{w}$ the measurement noise vector. The state variables that minimize the estimation error can be calculated by setting the error function derivatives to zero, i.e.

$$
\boldsymbol{H}_{x}^{T} \boldsymbol{R}^{-1}[\boldsymbol{z}-\boldsymbol{h}(\boldsymbol{x}, \boldsymbol{u}, \boldsymbol{w})]=0
$$

Where $\boldsymbol{H}_{\boldsymbol{x}}$ is the Jacobian matrix of $\boldsymbol{h}$ respect of $\boldsymbol{x}, \boldsymbol{H}_{x}=[\partial \boldsymbol{h} / \partial \boldsymbol{x}]$, the order of $\boldsymbol{H}_{x}$ is $m \times n$. $\boldsymbol{R}$ is the measurement covariance matrix. Equation (23) is a set of nonlinear equations that can be solved using iterative numerical methods, e.g., Newton-Raphson. The Jacobian of (23) is:

$$
\boldsymbol{J}(\boldsymbol{x})=\boldsymbol{H}_{x}^{T}(\boldsymbol{x}) \boldsymbol{R}^{-1} \partial[\boldsymbol{z}-\boldsymbol{h}(\boldsymbol{x}, \boldsymbol{u}, \boldsymbol{w})] / \partial \boldsymbol{x}=-\boldsymbol{H}_{x}^{T}(\boldsymbol{x}) \boldsymbol{R}^{-1} \boldsymbol{H}_{x}(\boldsymbol{x})
$$

In particular, for the Newton-Raphson method, the iteration is defined by:

$$
\left[\boldsymbol{H}_{\boldsymbol{x}}^{T}\left(\boldsymbol{x}_{k}\right) \boldsymbol{R}^{-1} \boldsymbol{H}_{\boldsymbol{x}}\left(\boldsymbol{x}_{k}\right)\right]\left[\boldsymbol{x}_{k-1}-\boldsymbol{x}_{k}\right]=\boldsymbol{H}_{\boldsymbol{x}}^{T}\left(\boldsymbol{x}_{k}\right) \boldsymbol{R}^{-1}\left[\boldsymbol{z}_{k}-\boldsymbol{h}\left(\boldsymbol{x}_{k}, \boldsymbol{u}_{k}, \boldsymbol{w}_{k}\right)\right]
$$

This iterative expression can be solved repeatedly using LU factorization, converging to obtain $\boldsymbol{x}_{k+1}$, which is equal to the state that minimize the state estimation error of (22). The procedure from (22) to (25) must be applied each time step during the time-domain analysis. When the system is underdetermined or ill-conditioned, the solution cannot exist or the state estimation error is considerable, under this condition an alternative is to apply the singular value decomposition (SVD) procedure.

The singular value decomposition (SVD) obtains a unique estimated solution if the system is over or normal-determined, $m>n$ or $m=n$, respectively. When the system is under-determined, $m<n$, which is the examined case in this paper, the SVD obtains an estimated solution with minimum norm and defines the system observability [33-35]. The nonlinear measurement model must be linearized using the Taylor's formula about an initial condition of the states $\boldsymbol{x}_{0}$, i.e.

$$
\boldsymbol{z}=\boldsymbol{h}\left(\boldsymbol{x}_{0}, \boldsymbol{u}_{0}, \boldsymbol{w}_{0}\right)+\left[\left.\frac{d \boldsymbol{h}(\boldsymbol{x}, \boldsymbol{u}, \boldsymbol{w})}{\boldsymbol{d x}}\right|_{\boldsymbol{x}=x_{0}}\right]\left(x-x_{0}\right)+\ldots
$$

If only first two terms are represented, the linear term is written as $\boldsymbol{H}\left(\boldsymbol{x}-\boldsymbol{x}_{0}\right)$, and the measurement equation can be represented as $\boldsymbol{z}=\boldsymbol{H} \boldsymbol{x}$. SVD factorizes the linearized measurement matrix $\boldsymbol{H}=\boldsymbol{U} \boldsymbol{W} \boldsymbol{V}^{T}$; if the system is over or normal-determined, the state estimation is assessed using the SVD as,

$$
\boldsymbol{x}=\boldsymbol{V} \boldsymbol{W}^{-1} \boldsymbol{U}^{T} \boldsymbol{z}
$$

If $\boldsymbol{H}$ is ill-conditioned or under-determined, SVD gives a solution using the pseudo-inverse. The division of the largest to the smallest singular value gives the condition number. A matrix with a large condition number is ill-conditioned. If a singular value is zero or near zero, a zero is placed in the corresponding diagonal element of $\boldsymbol{W}^{-1}$ instead of $1 / w$, if, 


$$
\boldsymbol{W}=\left[\begin{array}{ll}
\boldsymbol{S} & 0 \\
0 & 0
\end{array}\right]
$$

The pseudo-inverse $\boldsymbol{W}^{+}$is defined by:

$$
\boldsymbol{W}^{+}=\left[\begin{array}{cc}
\boldsymbol{S}^{-1} & 0 \\
0 & 0
\end{array}\right]
$$

The pseudo-inverse of $\boldsymbol{H}$ is equal to $\boldsymbol{V} \boldsymbol{W}^{+} \boldsymbol{U}^{T}$ and the state estimation can be assessed using the pseudoinverse, i.e.

$$
\boldsymbol{x}=\boldsymbol{V} \boldsymbol{W}^{+} \boldsymbol{U}^{T} \boldsymbol{z}
$$

The time-domain state estimation can be assessed using the SVD each time step during the time interval under analysis, mainly when the system is under-determined, which is the condition to be considered in this contribution. The HSE obtained using the EKF is compared against the SVD state estimation, as shown in [34].

\section{CASE STUDIES}

Figure 2 shows the modified IEEE 14 bus test power system, analyzed in the next case studies. A threephase base power of $100 \mathrm{MVA}$ and a line-to-line base voltage of $230 \mathrm{kV}$ are assumed. Appendix A gives the additional component data.

Transmission lines 1-2, 1-5, 2-3, 2-4, 2-5, 3-4 and 4-5 are represented by a PI equivalent model and the rest of lines by series impedance; the transformers 4-9, 5-6, 4-8-9, are represented by a leakage reactance, according to the IEEE 14 bus test system [36]. The generators are individually represented by a sinusoidal voltage source model. 


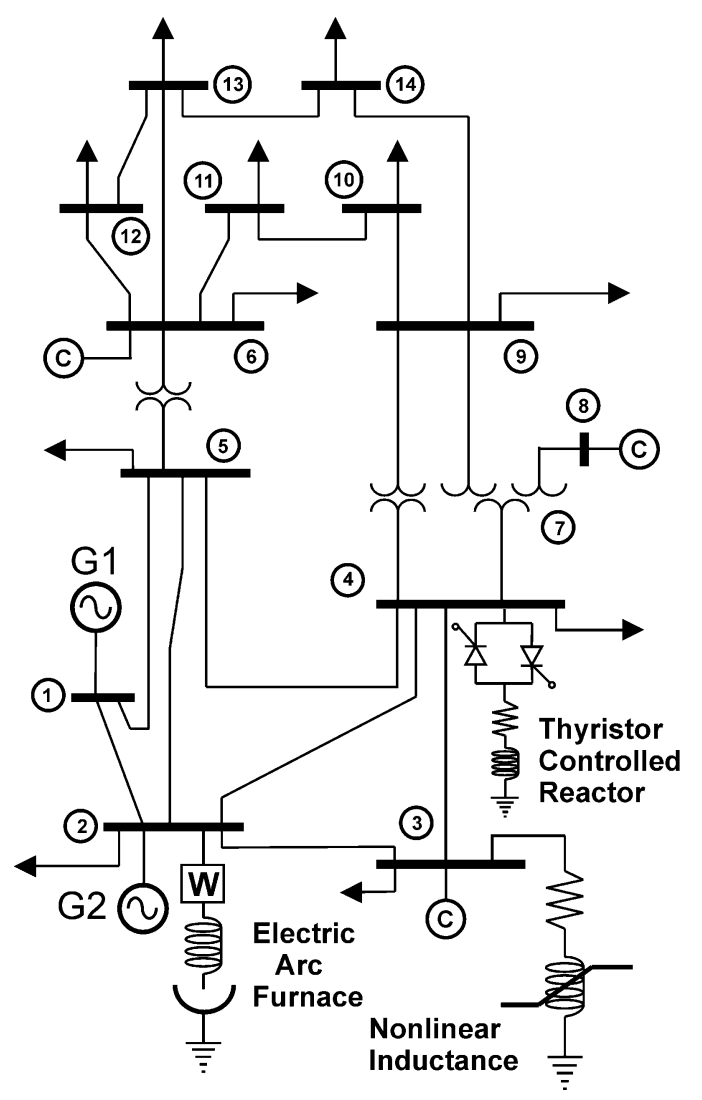

Figure 2. Modified IEEE 14 bus test power system with nonlinear loads.

Nonlinear loads are connected to nodes 2, 3 and 4; electric arc furnace (EAF), nonlinear inductance and thyristor controlled reactor (TCR), respectively, as shown in Figure 2. These loads inject harmonic currents, generating current and voltage distortion [37-38]; under this load condition the HSE is evaluated using the EKF algorithm. Appendix B details the nonlinear load modelling.

A set of 47 differential equations is to be solved for a single-phase analysis, representing the system model (1). The EKF-HSE is applied with 40 measurements synchronously taken from the system to form the measurements state estimation equation (5) with an under-determined condition. These measurements are 20 line currents, 14 nodal voltages, 5 load currents and the EAF real power.

Equations (4) and (5) are solved to obtain the estimated state and output variables using the EKF algorithm; i.e. equations (13)-(21). Appendix C gives the definition of $\boldsymbol{\Phi}$ and $\boldsymbol{H}$ matrices, these matrices are evaluated each time step using functions defined with the Jacobians of $\boldsymbol{f}$ and $\boldsymbol{h}$, respectively. Tables 1 and 2 present the state variables and the output variables to be monitored respectively. The state variables of load currents 40-43, the nonlinear inductance flux and current, and the TCR current are unmonitored; these state variables are only estimated. 
The HSE is evaluated using 512 points per cycle, with a time step of 32.55 microseconds, the fundamental frequency is $60 \mathrm{~Hz}$, the initial condition is set to zero, except for the EAF radius that is set to 0.1 p.u.; the initial state covariance matrix $\boldsymbol{P}_{0}$ is a diagonal matrix with elements equal to 1.5 ; the process and measurement noise are defined with normal distribution, zero mean and a standard deviation of 0.01 . The process and measurement covariance matrices $\boldsymbol{Q}$ and $\boldsymbol{R}$, are diagonal matrices with 0.01 elements of $n \times n$ and $m \times m$ order respectively; $n=47$ states and $m=40$ measurements. The measurements matrix $\boldsymbol{H}, m \times n$ order, yields in this case to an under-determined condition.

\subsection{Nonlinear Harmonic State Estimation using the Extended Kalman Filter}

Figure 3 shows the actual and estimated state variables and their differences for all the load currents; load current state variables 33-39 are monitored and load current state variables 40-43 are unmonitored, they are only estimated. Please observe that a close agreement is obtained between the actual and the proposed EKF method responses; the NRMSE is of $1.7 \%$ during the state estimation interval. The same case is solved using the KF; the result is compared with the actual values, the NRMSE is of 3.6\%.

When the estimated state variables are known, other variables can be calculated to obtain the global system state. The actual values are obtained from the harmonic propagation solution using the set of differential equations that models the system and the fourth-order Runge-Kutta method to compare and validate the EKF estimation. A small-scale test system has been used to illustrate the application of the proposed dynamic state estimation methodology. However, it can be applied to the practical assessment of large-scale power networks. The EKF method can appropriately account for the presence of high frequency effects by using the adequate step size during the time-domain solution, which can have an implicit harmonic content of low and high frequencies. 
(a) Load currents - Actual

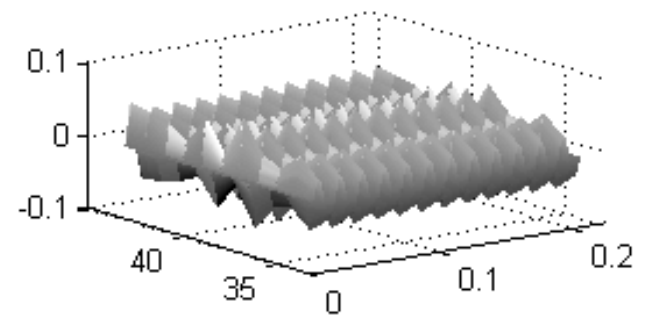

(b) EKF estimated

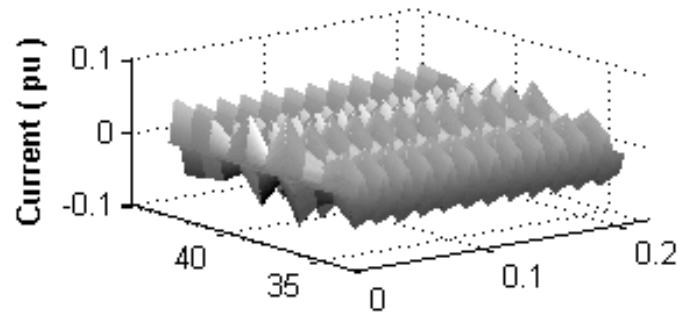

(d) Difference EKF

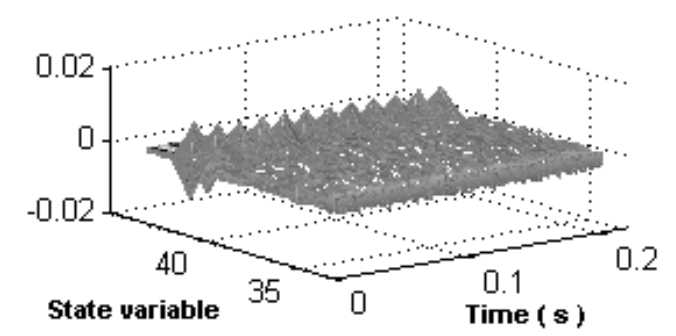

(c) KF estimated

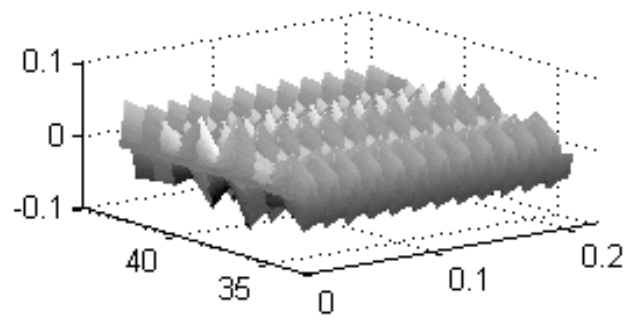

(e) Difference KF

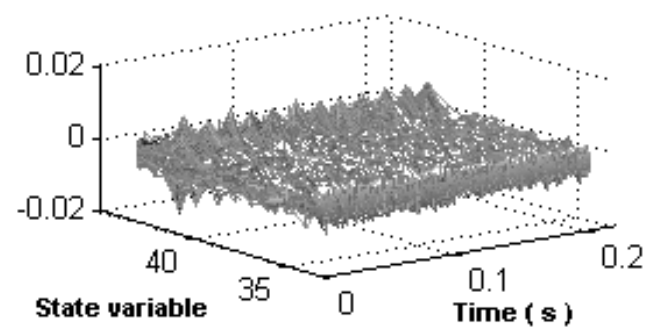

Figure 3. Load currents, state variables 33-43. (a) Actual, (b) EKF estimate, (c) KF estimate, (d) Difference EKF and (e) Difference KF.

Figure 4 shows the current waveforms of lines 12-13 and 13-14. These are the most distorted currents in the system, a close agreement is observed between the actual and the proposed EKF method which present a total harmonic distortion (THD) of 105.8\% and 91.5\% for the current in lines 12-13 and 13-14 respectively [39], as shown in Figure 4(a)-(c). The differences observed are mainly due to the noisy condition in the measurements, i.e. a noise of $1 \%$ has been assumed. The harmonic distortion is due to harmonic injection originated by the nonlinear loads connected at nodes 2, 3 and 4, i.e. the EAF at node 2, the nonlinear inductance at node 3 and the TCR at node 4, as illustrated in Figure 2 [40]. The maximum estimation error is of $0.4 \%$ for current line $12-13$ and of $0.35 \%$ for current line $13-14$, as shown in Figure 4(b). The NRMSE is of $0.65 \%$ for current line $12-13$ and of $0.52 \%$ for current line 13 14. 
(a) Line Currents Line 12-13

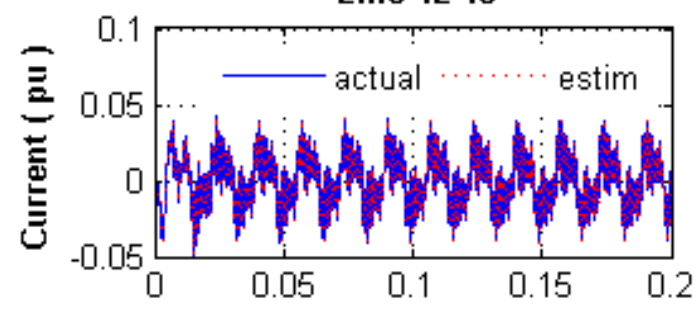

(b) State Estimation Error

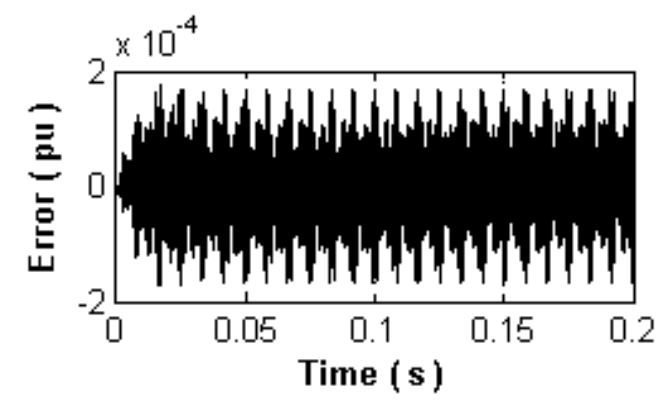

(c) Harmonic Spectrum

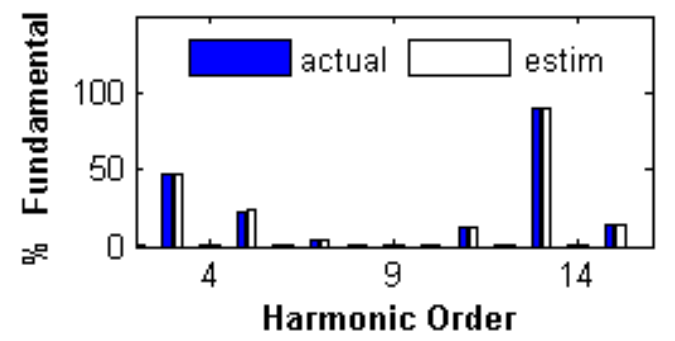

Line 13-14
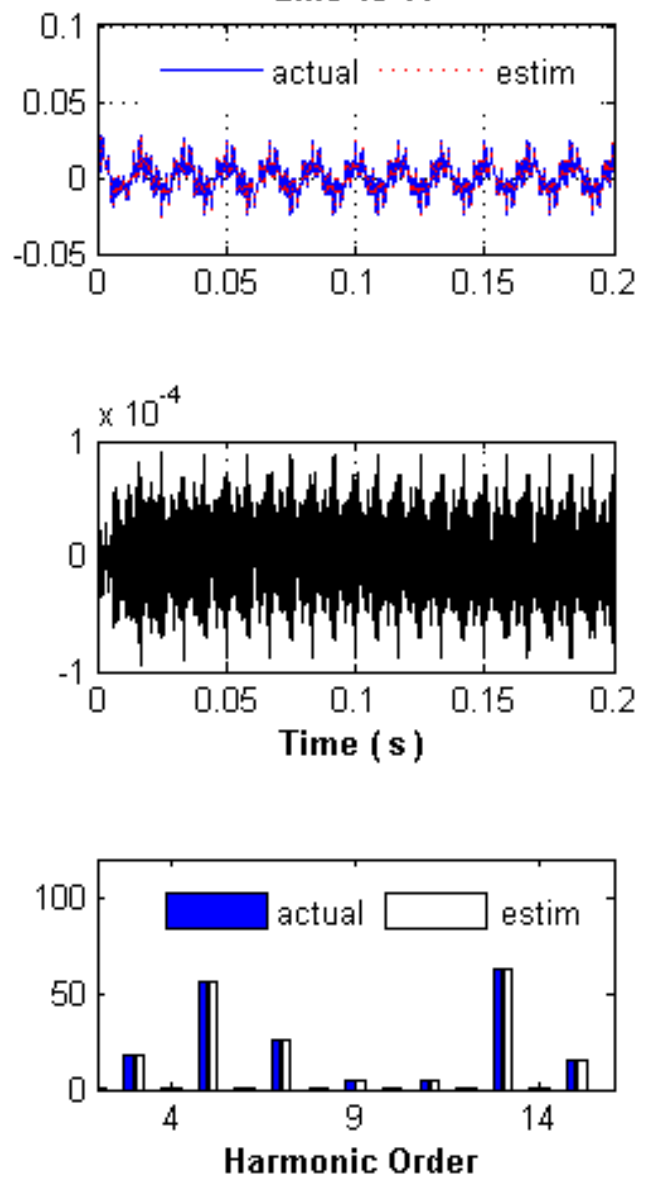

Figure 4. Line currents. (a) Actual, EKF estimate line currents 12-13 and 13-14, (b) Estimation error and (c) Harmonic spectrum.

Figure 5 shows the estimated waveform for the load current at bus 14 of the IEEE 14 bus test system, obtained using the EKF, the KF and the SVD. A close agreement is present between the waveforms but with a different state estimation error or residual. The NRMSE is assessed using the actual data and the estimated results of each method. For this load current, the NRMSE is $1.7 \%$ for the EKF, $2.2 \%$ for the KF and $2.5 \%$ for the SVD. The EKF method assesses two Jacobians for $\boldsymbol{\Phi}$ and $\boldsymbol{H}$ matrices, equations (15) and (18), respectively, and an inverse matrix, equation (19). In this nonlinear case, the KF method applies the Taylor's formula for linearization and executes the ordinary KF algorithm, while the SVD method applies the Taylor's formula, assesses a Jacobian (26), performs the SVD decomposition and obtains an inverse matrix (29), as its main process steps. 
(a) Load current bus 14
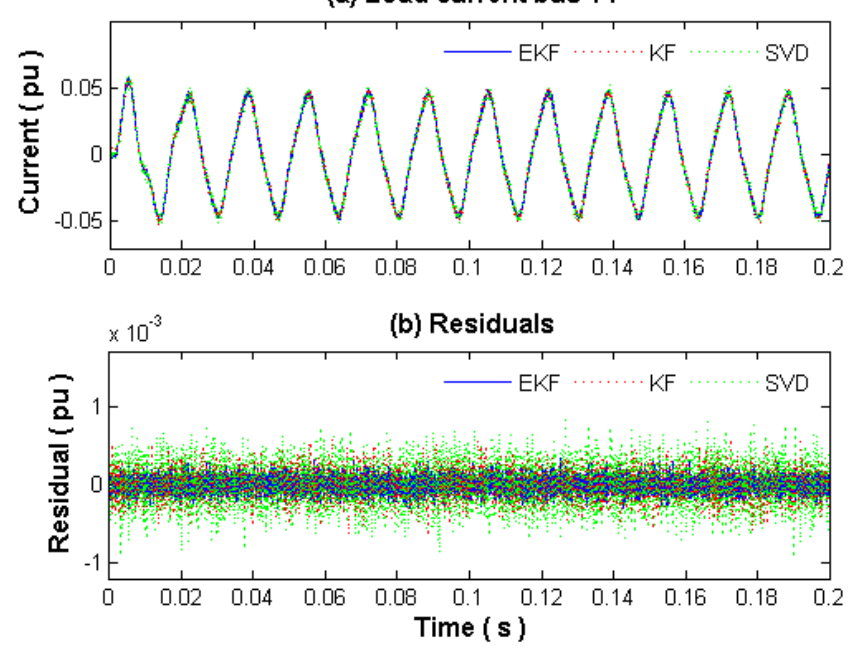

Figure 5. Load current at bus 14. (a) EKF, KF and SVD state estimation and (b) Residuals.

The nonlinear inductance current is shown in Figure 6; actual and EKF estimated waveforms closely agree, except for the first period. This current is evaluated with (B.2), and the parameters are indicated in Appendix A. This is an estimated variable, the state variable 44 (nonlinear inductance magnetic flux $\lambda$ ) cannot be monitored. The response obtained with the proposed EKF method closely matches the actual current waveform; they only differ during the first cycle of the initial transient; THD is $7.2 \%$.
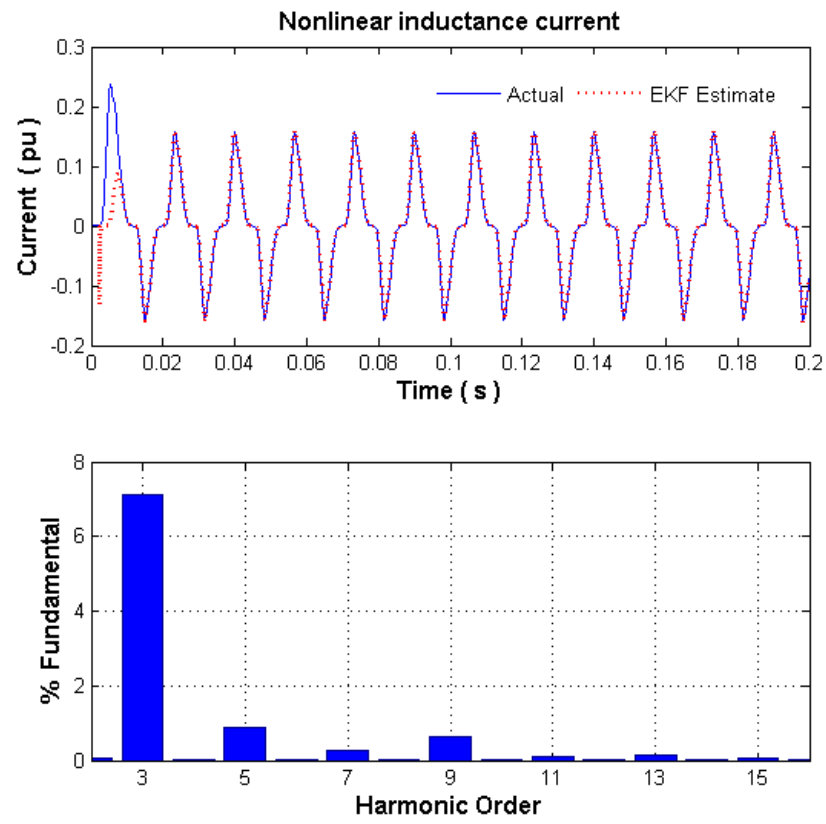

Figure 6. Nonlinear inductance current. Actual, EKF estimate and harmonic spectrum. 
The TCR current is shown in Figure 7, the actual and EKF estimated waveforms again closely agree during the interval of study. The TCR current, state variable 47 is only estimated as it is unmonitored. The harmonic content illustrates the harmonics injected by the TCR; the THD is $45.1 \%$ for actual and estimated waveforms; the TCR firing angle is 100 degrees.
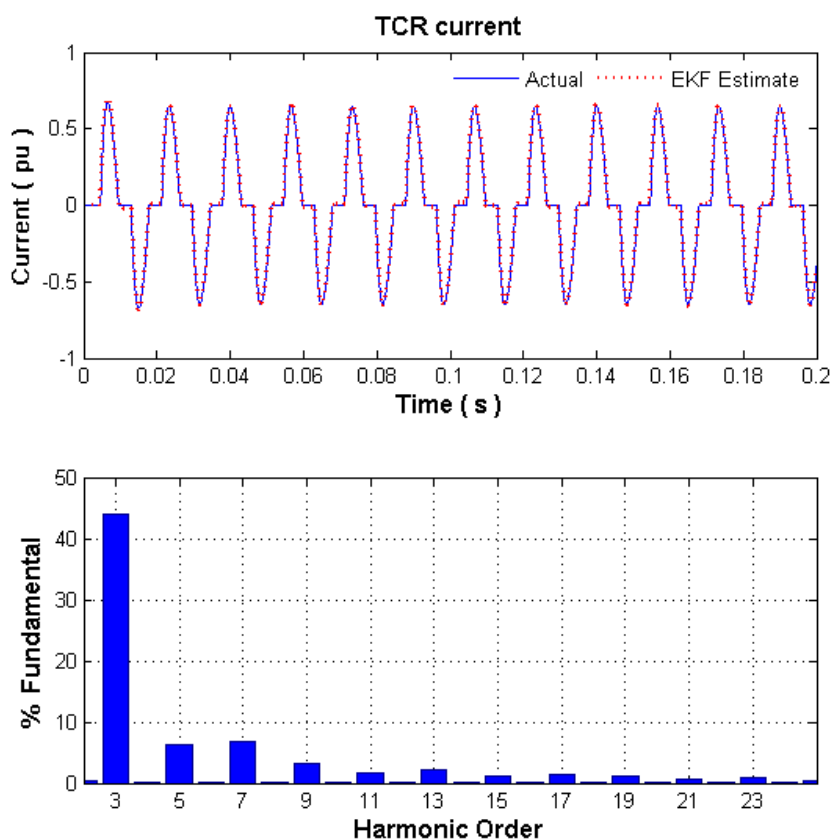

Figure 7. TCR current. Actual, EKF estimate and harmonic spectrum.

Figure 8 illustrates the current, radius and real power of the EAF, actual and EKF estimate for the initial transient of 12 cycles; EAF current has a dc component due to the EAF initial period. The arc radius and real power have high frequency components, which eventually disappear once the periodic steady state is achieved. However, they can be generated according to the EAF operation, originating harmonic currents. During this initial period, the actual and EAF estimated values overlap, by definition of (1) and (2), the EAF current and arc radius are state variables, while the real power is an output measured variable but not a state variable. The arc radius cannot be measured neither defined as measurement. Appendix B details the EAF model. 

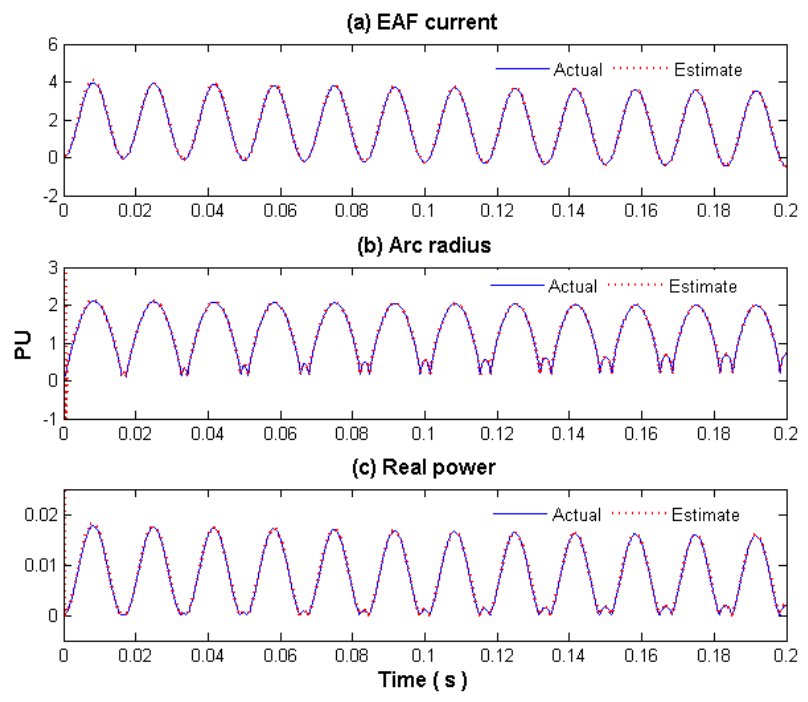

Figure 8. Actual and estimate EAF waveforms. (a) EAF current, (b) Arc radius and (c) Real power.

Figure 9 presents the fluctuations of the error covariance matrix $\boldsymbol{P}$ for the magnetic flux in the nonlinear inductance, the EAF current, the EAF arc radius and the TCR current. The magnetic flux at the nonlinear inductance varies according with the fluctuations of the EAF and TCR; the EAF current and arc radius vary according with the EAF real power fluctuations and the TCR current varies mainly according with the commutation of this load. Initially the error covariance $\boldsymbol{P}$ presents an initial transient of different duration for each element, eventually reaching a periodic steady state with a reduced state estimation error.
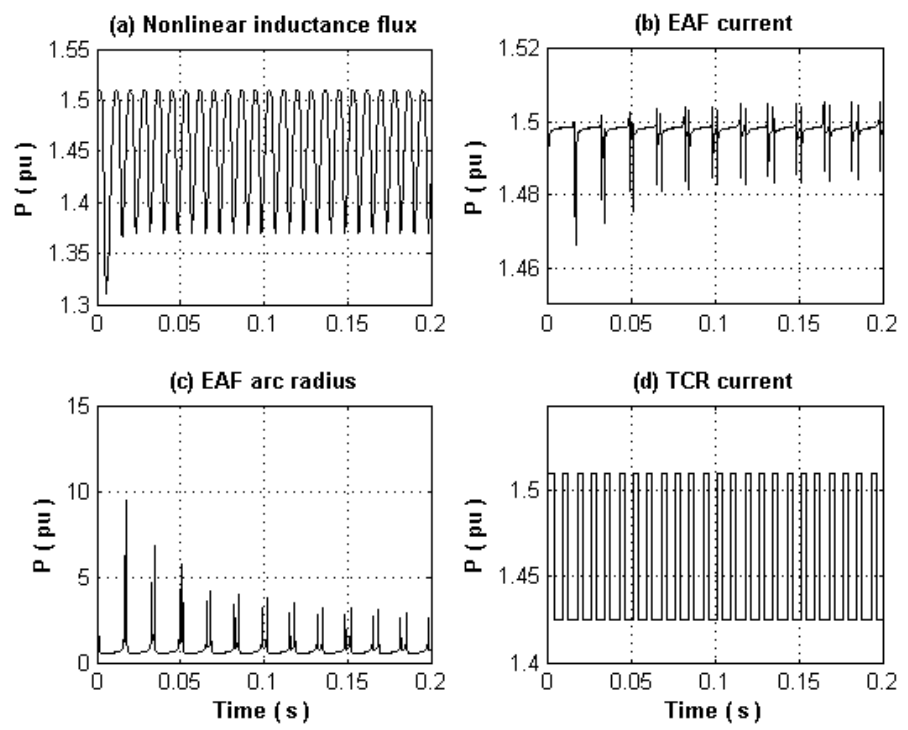

Figure 9. Error covariance $(\boldsymbol{P})$ during the time-domain estimation for state variables of nonlinear components. (a) Nonlinear inductance flux, (b) EAF current, (c) EAF arc radius and (d) TCR current. 


\subsection{Increase of measurement noise}

The EKF and KF are applied under a more noisy measurement environment than in the previous case study, where the noise was of $1 \%$. For this case study, the noise is increased to $4 \%$, which is added to the measurements. The EKF and KF are applied under identical load conditions, assuming the same topology for the system. Figure 10 illustrates the state estimation for the load currents in the power system. As it can be observed, the difference between the actual and estimated values increases with this noisy condition, mainly when the initial transient is present, after this, the error decreases when the system gradually settles to its periodic steady state. The NRMSE between the actual and the EKF state estimation is $3.19 \%$ and between the actual and the KF state estimation $6.33 \%$. This result is due mainly to the linearization process of the model to apply the KF.

(a) Load currents - Actual

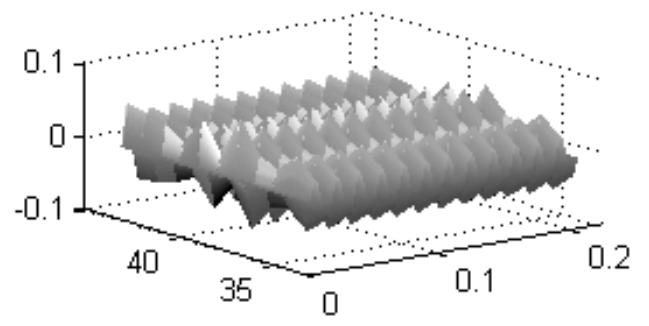

(b) EKF estimated

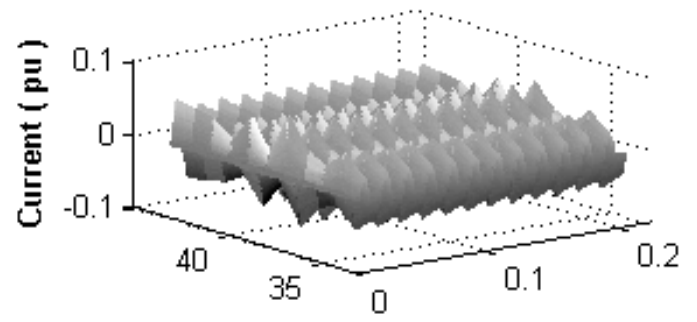

(d) Difference EKF

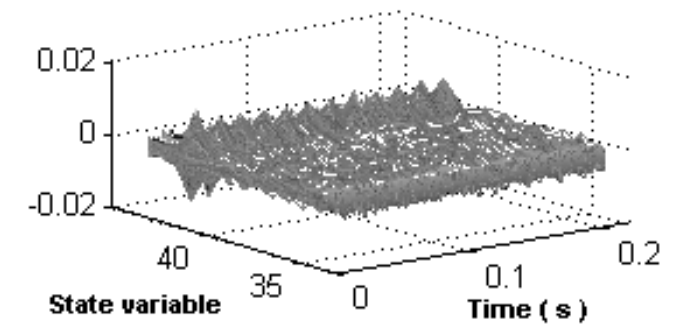

(c) KF estimated

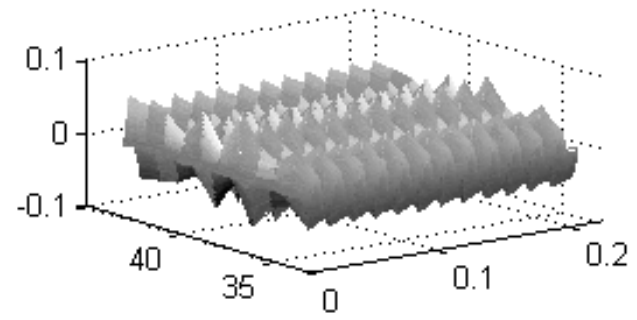

(e) Difference KF

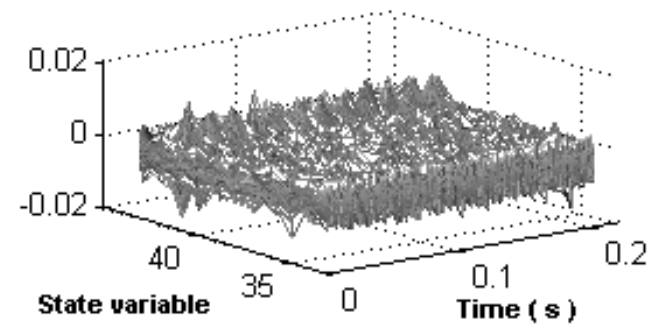

Figure 10. Load currents with a noise increase in measurements of 4\%, state variables 33-43. (a) Actual, (b) EKF estimate, (c) KF estimate, (d) Difference EKF and (e) Difference KF. 
Figure 11 shows the TCR current to analyze and compare the 1\% and 4\% noise conditions. Figure 11(a) shows that the waveform is not affected, but the difference increases with the noise, mainly during the TCR thyristor switching, due to the system changing condition, as shown in Figure 11(b). This difference is the state estimation error; the maximum error is $6.25 \%$ at the instant of commutation for $4 \%$ of noise, and $1.7 \%$ for $1 \%$ of noise. Figure 11(c) shows that during most of the time, the error is kept under $1.5 \%$ for both noise conditions. The NRMSE is of $0.82 \%$ for $1 \%$ of noise and of $2.15 \%$ for $4 \%$ of noise.

(a) TCR Current
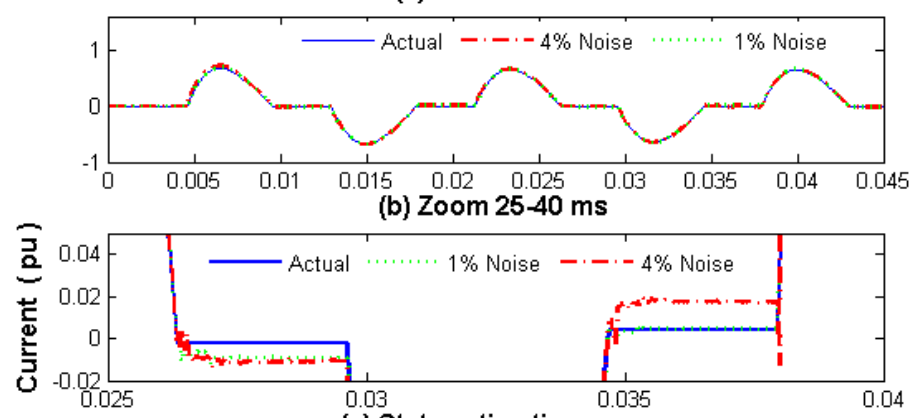

(c) State estimation error

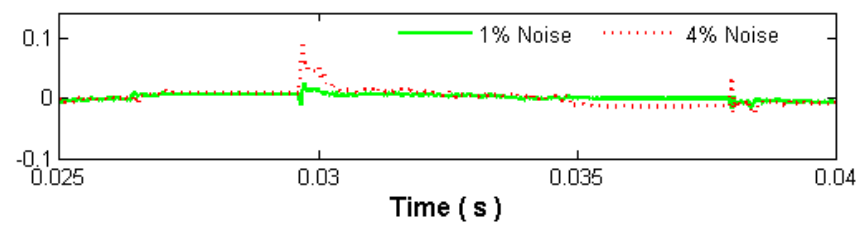

Figure 11. TCR current. (a) Actual, EKF estimate with 1 and 4\% of noisy measurements, (b) Zoom of TCR current, 25-40 ms. (c) TCR current state estimation error.

\subsection{Variation of sampling frequency}

Figure 12 describes the state estimation error in the TCR current for three different conditions corresponding to the number of points per cycle (NPC), 256 (dotted-segment line), 512 (continuous line), and 1024 (dotted line), respectively, when the TCR firing angle is 100 degrees. The fourth order RungeKutta method was applied to solve the set of differential equations modelling the power system. High frequency variations are due to the thyristor commutation. After these variations, there is an error reduction, i.e. of the order of $1.6 \%$. The state estimation error decreases when the number of points per cycle is increased; however, the sampling frequency must be increased. With more points to process numerically, the computational effort is increased to assess the EKF state estimation. There must be a balance between the computational effort and the number of points per cycle which defines the sampling frequency of the waveforms. 


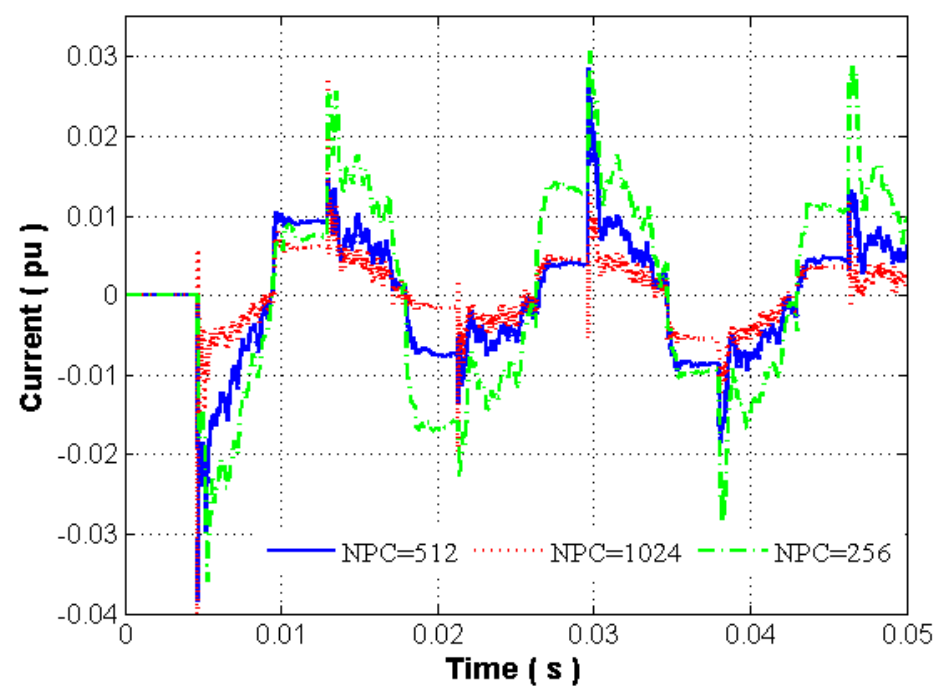

Figure 12. TCR current state estimation error, actual minus estimate values with different sampling frequencies, NPC number of points per cycle.

Table 3 gives the sampling frequency and the execution time of the state estimation as a function of the NPC; these frequencies can be practically implemented with the actual measurement and instrumentation technology. The execution time increases as the NPC increases, however, the state estimation results are more accurate.

\subsection{Harmonic state estimation using the IEEE 30-bus test power system with nonlinear loads}

The proposed methodology for harmonic state estimation through the EKF is applied to the IEEE 30-bus test power system modified with nonlinear electrical loads. An EAF is connected to node 2, a nonlinear inductance to node 5 and a TCR to node 6 . Reference [36] gives the test system data. The network model has 110 states with 41 line currents, 30 nodal voltages, 6 generator currents, 29 load currents and 4 states for the nonlinear inductance, the EAF and the TCR; 110 differential equations are to be solved for a single-phase analysis, modelling the power system by (1).

The measurement equation (5) is defined to obtain an observable condition for the state variables, in this case 103 measurements to assess 110 state variables; each measurement is associated with its corresponding state variable. The measurements are obtained from the time-domain simulation of the test power system. This simulation is evaluated using the fourth-order Runge-Kutta method. Noise randomly generated is added to the measurements, the EKF gives the HSE solution.

Figure 13 shows the results of the HSE using EKF and KF for line currents. The measurements are added with a noise of $2 \%$. The differences are appreciable only during the first cycle when the initial 
transient is present; the NRMSE is $1.94 \%$ for the EKF and $4.7 \%$ for the KF, for the state variable 3, which presents the largest state estimation difference.

(a) Line currents - Actual

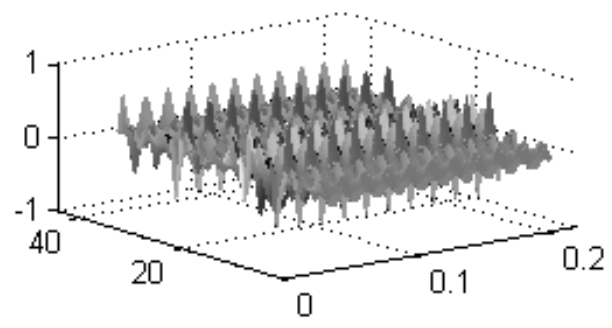

(b) EKF estimated

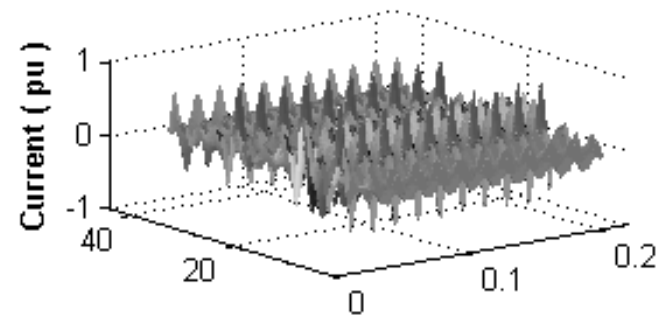

(d) Difference EKF

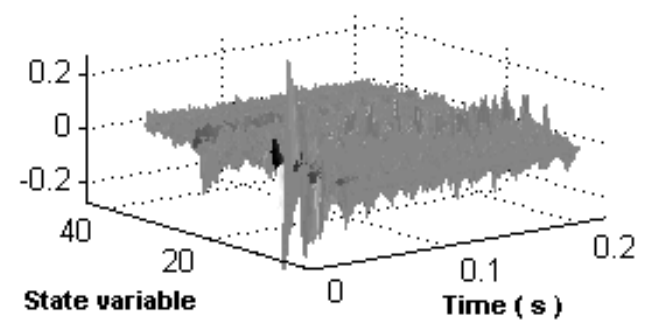

(c) KF estimated

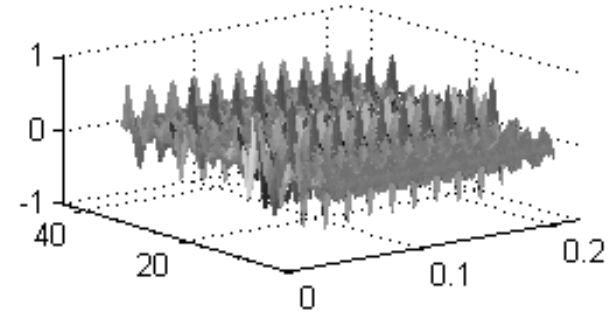

(e) Difference KF

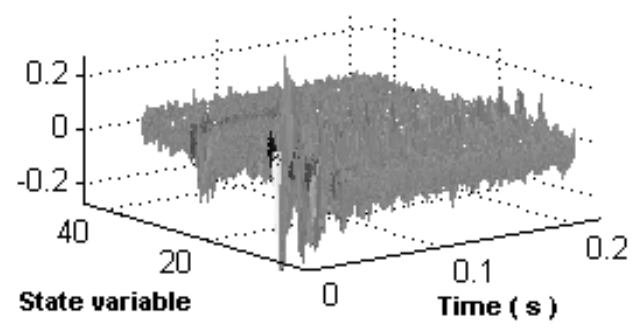

Figure 13. Line currents, state variables 1-41, IEEE 30-bus test system with nonlinear loads. (a) Actual, (b) EKF estimate, (c) KF estimate, (d) Difference EKF and (e) Difference KF.

Figure 14 presents the actual and estimated waveforms for the currents in lines 3-4 and 6-7. These are the most distorted currents. THD is of 51.9\% and 36.7\% for lines 3-4 and 6-7 respectively. The state estimation error is less than 2\% for line 3-4 and less than $1 \%$ for line 6-7. The actual and estimated harmonic spectra agree in both line currents with a maximum error of $1.8 \%$. 
(a) Line Currents

Line 34

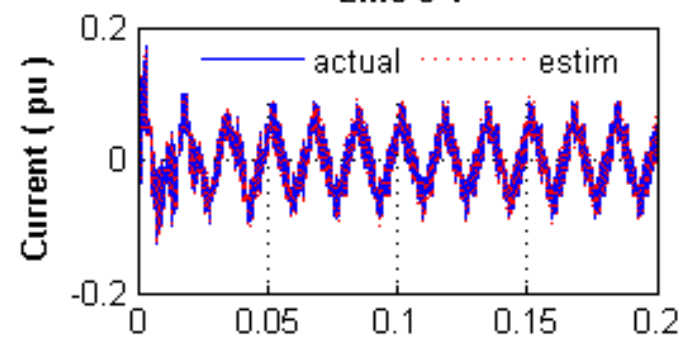

(b) State Estimation Error

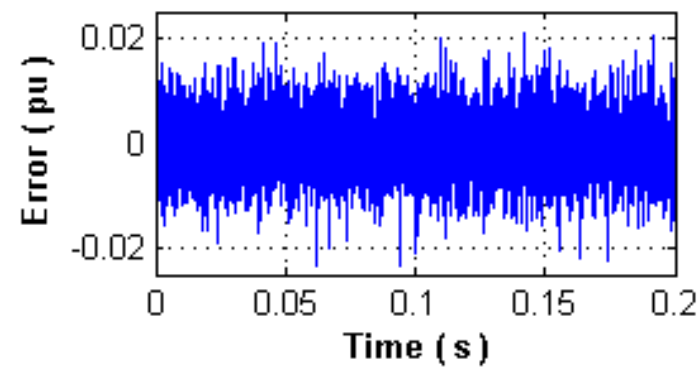

(c) Harmonic spectrum

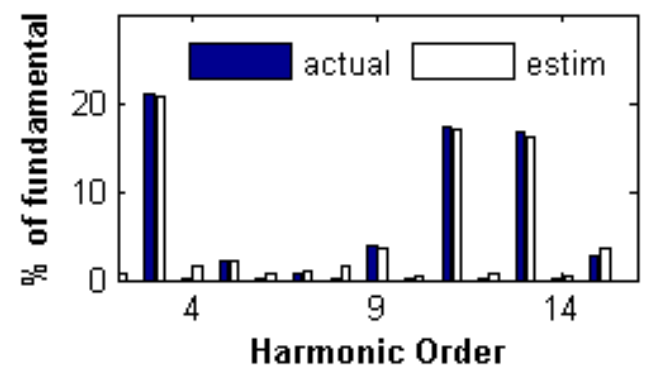

Line 6-7
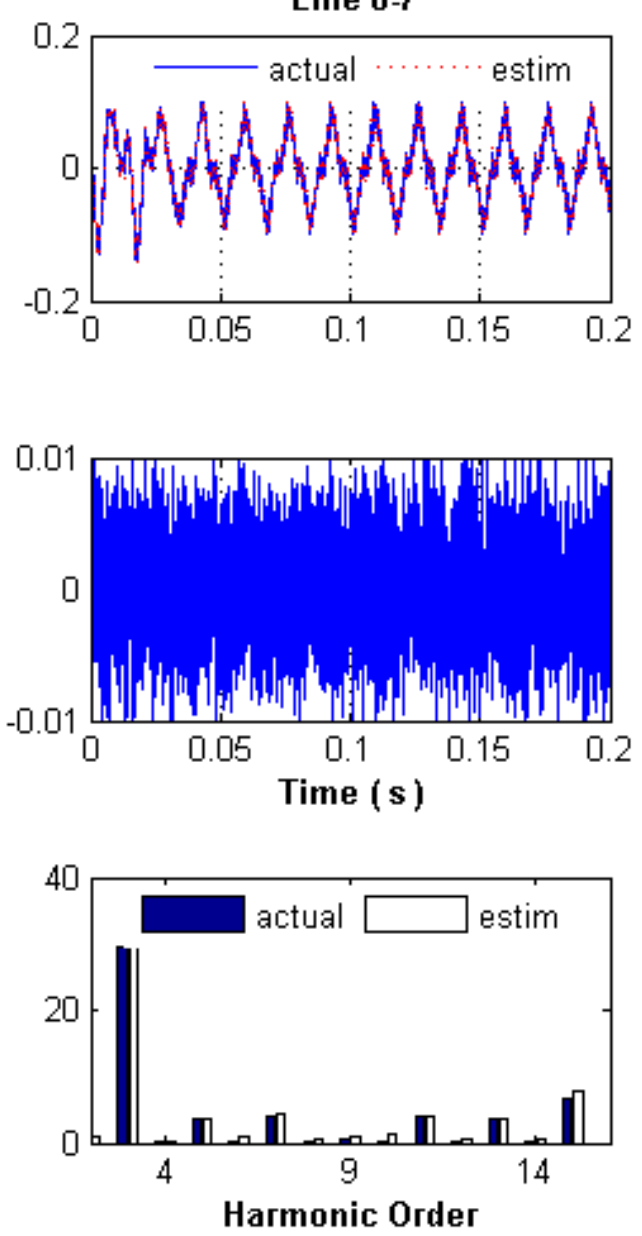

Figure 14. Line currents 3-4 and 6-7 for the IEEE 30-bus test system. (a) Actual and EKF estimate, (b) Estimation error and (c) Harmonic spectrum.

Figure 15 shows the estimated waveform using the EKF for the load current at bus 30 of the IEEE 30 bus test system and the estimated waveforms by the KF and the SVD methods. A close agreement is again obtained between the EKF, KF and SVD estimated waveforms. The NRMSE is evaluated using the actual data and the estimated results. For this load current, the NRMSE is $1.7 \%$ for the EKF, $2.2 \%$ for the KF and $2.6 \%$ for the SVD. 

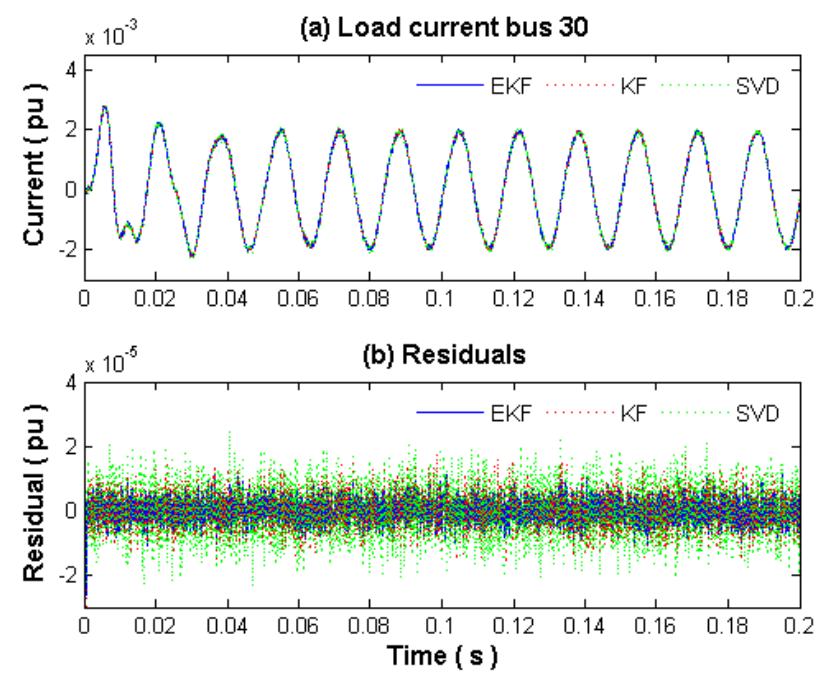

Figure 15. Load current at bus 30. (a) EKF, KF and SVD state estimation and (b) Residuals.

The time-domain HSE case studies were implemented with an Intel(R) Core(TM) 2 Duo CPU T5870, 2.0 GHz, 2.84 GB RAM, 32 bits, using the Matlab script language. As an extension of this work, it is expected that the computer effort can be considerably decreased with the application of efficient computational techniques such as the parallel processing and compiled files.

\section{CONCLUSIONS}

A time-domain harmonic state estimator based on the application of the EKF has been proposed. The EKF results have been successfully compared against the actual power system harmonic response.

The power system has been mathematically modeled by a set of nonlinear differential equations. The harmonic flows in the system depend on the sources, their location in the system, the network topology and the nonlinear loads.

The waveforms of the estimated variables have been obtained with the proposed time-domain EKFHSE method and their harmonic content evaluated with the discrete Fourier transform.

The state estimation error is inversely proportional to the number of points per cycle but the computational effort to evaluate the state estimation is proportional to this number. The error is proportional to the noise; i.e. for moderated noise, the error is kept low, on average below 1\%, mainly in periodic steady state. For an assumed noise of $4 \%$ the error was on average of $1.5 \%$ being higher at the start of the simulation due to the initial transient condition of the system.

The proposed HSE method using the EKF requires the power system model and a set of synchronized measurements from the system to estimate the state variables, then the estimated output variables can be 
assessed to be compared with the measured output variables and finally to obtain the state estimation error

\section{LIST OF SYMBOLS AND ABBREVIATIONS}

\subsection{Symbols}

$\boldsymbol{e} \quad$ state estimation error

$f \quad$ nonlinear state function

$\boldsymbol{h}$ nonlinear output function

$\boldsymbol{i} \quad$ instantaneous current

$k \quad$ time instant $t=k \Delta t$

$k-1 \quad$ time instant $t=(k-1) \Delta t$

$m \quad$ number of measurements

$n \quad$ number of state variables

$t$ time

$\boldsymbol{u}$ input vector

$\boldsymbol{v}$ process noise vector

$\boldsymbol{w}$ measurement noise vector

$\boldsymbol{x}$ state vector

$y \quad$ output vector

$\boldsymbol{z}$ measurement vector

E expected value

$\boldsymbol{H}$ measurements matrix

I unitary matrix

$\boldsymbol{K}$ extended Kalman filter gain

$\mathrm{N}$ normal distribution

$\boldsymbol{P}$ error covariance matrix

$\boldsymbol{Q}$ process noise covariance

$\boldsymbol{R}$ measurement noise covariance

$\boldsymbol{U}$ column-orthogonal matrix of SVD

$\boldsymbol{V}$ orthogonal matrix of SVD

$\boldsymbol{W}$ diagonal matrix with positive or zero singular values 
$+\quad$ a posteriori or after measurement estimate

- $\quad$ a priori or before measurement estimate

$\Delta t \quad$ step time

$\lambda \quad$ magnetic flux

$\sigma \quad$ standard deviation

Ф state transition matrix

\subsection{Abbreviations}

DFT Discrete Fourier Transform

EAF Electric arc furnace

EKF Extended Kalman Filter

HSE Harmonic State Estimation

MSE Mean square error

NRMSE Normalized root mean square error

NLS Nonlinear least squares

NPC number of points per cycle

PQSE Power Quality State Estimation

RTU Remote terminal unit

SVD Singular Value Decomposition

TCR Thyristor controlled reactor

THD Total harmonic distortion

TSE Transient State Estimation

VSSE Voltage Sag Estimation

\section{ACKNOWLEDGMENTS}

The authors gratefully acknowledge the Universidad Michoacana de San Nicolás de Hidalgo through the División de Estudios de Posgrado, Facultad de Ingeniería Eléctrica (DEP-FIE), Morelia, México, for the facilities granted to carry out this investigation. The authors wish to thank the Consejo Nacional de Ciencia y Tecnología of México (CONACYT) for the financial support received to develop this research work. 


\section{REFERENCES}

[1] Arrillaga J., Watson N.R., Chen S. Power system quality assessment, John Wiley \& Sons, 2001.

[2] Yu K.K.C., Watson N.R. An approximate method for transient state estimation. IEEE Trans. Power Del. 2007; 22:1680-1687. DOI: 10.1109/TPWRD.2007.901147

[3] Espinosa-Juarez E., Hernandez A. A method for voltage sag state estimation in power systems. IEEE Trans. Power Del. 2007; 22:2517-2526. DOI: 10.1109/TPWRD.2007.905587

[4] Bollen M.H.J., Ribeiro P., Gu I.Y.H., Duque C.A. Trends, challenges and opportunities in power quality research. European Transactions on Electrical Power 2010; 20:3-18. DOI: 10.1002/etep.370

[5] Pérez E., Barros J. An extended Kalman filtering approach for detection and analysis of voltages dips in power systems. Electric Power Systems Research 2008; 78:618-625.

DOI:10.1016/j.epsr.2007.05.006

[6] Heydt G.T. Electric power quality; 2nd edn, Stars in a Circle Publications, 1991.

[7] Dugan R.C., McGranaghan M.F., Santoso S., Wayne B.H. Electrical power systems quality; 2nd edn, McGraw-Hill, 2002.

[8] Grewal M.S., Andrews A.P. Kalman filtering: theory and practice using matlab; 2nd edn, John Wiley \& Sons, 2001.

[9] Elnady A., Salama M.M.A. Unified approach for mitigating sag and voltage flicker using the dstatcom. IEEE Trans. Power Del. 2005; 20:992-1000. DOI: 10.1109/TPWRD.2004.837670

[10] Dash P.K., Pradhan A.K., Panda G. Frequency Estimation of Distorted Power System Signals Using Extended Complex Kalman Filter. IEEE Trans. Power Del. 1999; 14:761-766.

DOI: $10.1109 / 61.772312$

[11] Aiello M., Cataliotti A., Cosentino V., Nuccio S. Synchronization Techniques for Power Quality Instruments. IEEE Transactions on Instrumentation and Measurement, 2007; 56:1511-1519.

[12] Van der Heijden F., Duin R.P.W., Ridder D., Tax D.M.J. Classification, parameter estimation and state estimation; John Wiley \& Sons, 2004.

[13] Hajimolahoseini H., Reza T.M., Soltanian-Zadeh H. Extended Kalman filter frequency tracker for nonstationary harmonic signals. Measurement, 2012; 45:126-132.

[14] Semlyen A., Medina A. Computation of the periodic steady state in systems with nonlinear components using a hybrid time and frequency domain methodology. IEEE Trans. Power Syst. 1995; 10:1498-1504. DOI: 10.1109/59.466497 
[15] Singh G.K. Power system harmonics research: a survey. European Transactions on Electrical Power 2009; 19:151-172. DOI: 10.1002/etep.201

[16] Tan T.L., Chen S., Choi S.S. An overview of power quality state estimation. 7th Int. IEEE power engineering conf., IPEC, 2005. DOI: 10.1109/IPEC.2005.206920

[17] Ghahremani E., Kamwa I. Simultaneous state and input estimation of a synchronous machine using the extended Kalman filter with unknown inputs. IEEE Int. Electric Machines \& Drives Conf., IEMDC, 2011, pp. 1468-1473. DOI: 10.1109/IEMDC.2011.5994825

[18] Wang G., Liu C., Bhatt N., Farantatos E., Patel M. Observability of nonlinear power system dynamics using synchrophasor data. International Transactions on Electrical Energy Systems, DOI: 10.1002/etep 2016, Aug. 2015.

[19] Rizwan Khan M., Iqbal A. Extended Kalman filter based speeds estimation of series-connected fivephase two-motor drive system. Simulation Modelling Practice and Theory 2009; 17:1346-1360. DOI:10.1016/j.simpat.2009.05.007

[20] Samantaray S.R., Dash P.K. High impedance fault detection in distribution feeders using extended kalman filter and support vector machine. European Transactions on Electrical Power 2010; 20:382-393. DOI: 10.1002/etep.321

[21] Ghahremani E., Kamwa I. Dynamic state estimation in power system by applying the extended Kalman filter with unknown inputs to phasor measurements. IEEE Trans. Power Syst. 2011; 26:2556-2566. DOI: 10.1109/TPWRS.2011.2145396

[22] Moreno V.M., Pigazo A. Kalman Filter: Recent Advances and Applications; I-Tech Education and Publishing KG, Vienna, Austria, 2009.

[23] Watson N.R. Power quality state estimation. European Transactions on Electrical Power 2010; 20:19-33. DOI: 10.1002/etep.357

[24] Carranza O., Figueres E., Garcerá G., Gonzalez L.G. Comparative study of speed estimators with highly noisy measurement signals for Wind Generation Systems. Applied Energy 2011; 88:805813. DOI:10.1016/j.apenergy.2010.07.039

[25] Beides H.M., Heydt G.T. Dynamic state estimation of power system harmonics using Kalman filter methodology. IEEE Trans. Power Del. 1991; 6:1663-1670. DOI: 10.1109/61.97705

[26] Kennedy K., Lightbody G., Yacamini R. Power system harmonic analysis using the Kalman filter. IEEE PES Gen. Meet. 2003; 2:752-757. DOI: 10.1109/PES.2003.1270401 
[27] Kamwa I., Srinivasan K.A. Kalman filter-based technique for combined digital estimation of voltage flicker and phasor in power distribution systems. European Transactions on Electrical Power 1993; 3:131-142. DOI: 10.1002/etep.4450030204

[28] Jinghe Z., Welch G., Bishop G. LoDiM: A novel power system state estimation method with dynamic measurement selection. IEEE PES Gen. Meet. 2011. DOI: 10.1109/PES.2011.6039686

[29] Medina A., Cisneros-Magaña R. Time-domain harmonic state estimation based on the Kalman filter Poincare map and extrapolation to the limit cycle. IET Gener. Transm. Distrib. 2012; 6:1209-1217. DOI: 10.1049/iet-gtd.2012.0248

[30] Brigham E. O. The Fast Fourier Transform and its Applications; Prentice Hall, 1988.

[31] Watson N., Arrillaga J. Power systems electromagnetic transients simulation; 2nd edn, IET Power and Energy Series 39, 2007.

[32] Crow M. Computational Methods for Electric Power Systems; CRC Press, 2003.

[33] Hernandez A., Espinosa-Juarez E., Castro R. M., Izzeddine M. SVD Applied to Voltage Sag State Estimation. IEEE Trans. Power Del. 2013; 28:866-874. DOI: 10.1109/TPWRD.2012.2218627

[34] Yu K.K.C., Watson N.R. Three-phase harmonic state estimation using SVD for partially observable systems. Proc. of the 2004 International Conference on Power Systems Technology POWERCON 2004; 1:29-34. DOI: 10.1109/ICPST.2004.1459961

[35] Press W.H., Teukolsky S.A., Vetterling W.T., Flannery B.P. Numerical Recipes in C. The Art of Scientific Computing; 2nd Ed., Cambridge University Press, 1997.

[36] IEEE 14 and 30 bus test systems, power systems test case archive, $<$ http://www.ee.washington.edu/research/pstca $>$ [accessed: 14/02/2016].

[37] Soliman S.A., Alammari R.A. Harmonic modeling of linear and nonlinear loads based on Kalman filtering algorithm. Electric Power Systems Research 2004; 72:147-155.

DOI:10.1016/j.epsr.2004.03.012

[38] Wang Y., Mazin H.E., Xu W., Huang B. Estimating harmonic impact of individual loads using multiple linear regression analysis. International Transactions on Electrical Energy Systems, DOI: 10.1002/etep 2109, Jun. 2015.

[39] Carvalho J.R., Duque C.A., Lima M.A.A., Coury D.V., Ribeiro P.F. A novel DFT based method for spectral analysis under time-varying frequency conditions. Electric Power Systems Research, 2014; 108:74-81. 
[40] Kumar Saini M., Kapoor R. Classification of power quality events - A review. Electrical Power and Energy Systems, 2012; 43:11-19.

[41] Acha E., Madrigal M. Power systems harmonics computer modelling and analysis; John Wiley \& Sons, 2001.

[42] Dick E.P., Watson W. Transformer models for transients studies based on field measurements. IEEE Trans on PAS 1981; 100:409-419. DOI: 10.1109/TPAS.1981.316870

[43] Chang G., Hatziadoniu C., Xu W., Ribeiro P., Burch R., Grady W.M., Halpin M., Liu Y., Ranade S., Ruthman D., Watson N., Ortmeyer T., Wikston J., Medina A., Testa A., Gardinier R., Dinavahi V., Acram F., Lehn P. Modeling devices with nonlinear voltage-current characteristics for harmonic studies. IEEE Trans. Power Del. 2004; 19:1802-1811.

DOI: $10.1109 /$ TPWRD.2004.835429

[44] Acha E., Semlyen A., Rajakovic N. A harmonic domain computation package for nonlinear problems and its application to electric arcs. IEEE Trans. Power Del. 1990; 5:1390-1397. DOI: $10.1109 / 61.57981$

[45] Baggini A. Handbook of Power Quality; John Wiley \& Sons, 2008.

[46] Mohan N., Undeland T.M., Robbins W.P. Power Electronics, Converters, Applications and Design; 3rd edn, John Wiley \& Sons, 2003.

\section{APPENDIX A}

\section{Per unit values of the IEEE 14-bus test power system parameters}

Generators: Vpeak=1.0, frequency $=60 \mathrm{~Hz}$.

Nonlinear inductance: resistance $R m=4.0$, inductance $L m=1.0, a=0, b=0.3, n l=5$.

EAF: inductance Leaf $=0.5, k 1=0.004, k 2=0.0005, k 3=0.005, m f=0.0, n f=2.0$. Initial condition of EAF radius $=0.1$.

TCR: resistance $R t c r=1.0$, inductance $L t c r=0.5$, firing angle $\alpha=100 \mathrm{deg}$.

Reference [36] gives the line data.

Table A.1 presents the electrical linear loads for each node.

\section{APPENDIX B}

\section{Nonlinear models}

B.1. Nonlinear inductance 
The magnetic saturation generates harmonic currents in transient and steady state. This effect can be approximately modelled by an $n l$ order polynomial [41-42]. Figure B.1 illustrates a nonlinear inductor.

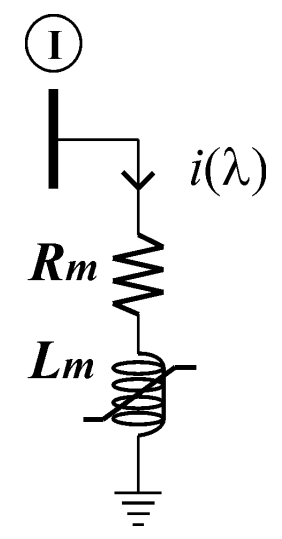

Figure B.1. Nonlinear inductor.

According to KVL,

$$
d \lambda / d t=v_{\mathrm{I}}-R_{m} i(\lambda)
$$

The nonlinearity is represented by the $i(\lambda)$ function, the magnetic saturation effect can be represented by the polynomial approximation, as,

$$
i(\lambda)=a \lambda+b \lambda^{n l}
$$

Where $n l$ is an odd number due to the odd symmetry of (B.2). Coefficients $a, b$ and $n l$ are chosen to fit the magnetic saturation curve. This nonlinearity can be also modeled by rational fraction or hyperbolic approximations [41-42].

\section{B.2. Electric arc furnace}

The EAF electrical distortion is an important issue due to its common use and the required power level. The highest current distortion is during the melting period [41]. Figure B.2 presents the EAF model.

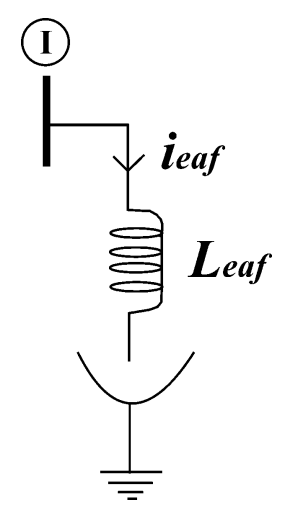

Figure B.2. Electric arc furnace. 
The electric arc model can be expressed by two differential equations based on the energy conservation. The electric arc power balance equation is,

$$
P_{1}+P_{2}=P_{3}
$$

$P_{1}$ is the heat power going to the environment, $P_{2}$ is the power to increase the internal energy arc affecting its radius, and $P_{3}$ is the total electric arc power converted into heat [42].

The EAF cooling effect is considered depending only of the electric arc radius $r_{e a f}$, as,

$$
P_{1}=k_{1} r_{\text {eaf }}^{n f}
$$

$P_{1}$ also depends of the arc temperature, but this dependence is less significant and therefore ignored, to keep a simple model. $n f=0$ if the arc cooling do not depend on its radius when the environment is hot. When the arc is long, the cooling area is its lateral surface, in this case $n f=1$. When the arc is short, the cooling is proportional to cross-section arc at the electrodes, then $n f=2$ [41], [43].

$P_{2}$ is proportional to the derivative of the electric arc energy, this energy is proportional to the square of the EAF radius,

$$
P_{2}=k_{2} r_{e a f}\left(d r_{e a f} / d t\right)
$$

Applying the ohm's law and the arc column resistivity, the electric arc voltage is

$$
v_{\text {eaf }}=\left(k_{3} r_{\text {eaf }}^{(-m f-2)}\right) i_{\text {eaf }}
$$

The arc column resistivity is inversely proportional to $r_{\text {eaf }}^{m f}$, where $m f=0 \ldots 2$, to consider that the electric arc may be hotter in the interior if it has a larger radius [44]. The total power of the EAF is,

$$
P_{3}=v_{\text {eaf }} i_{\text {eaf }}=\left(k_{3} r_{\text {eaf }}^{(-m f-2)}\right) i_{\text {eaf }}^{2}
$$

Substitution of (B.4), (B.5) and (B.7) into (B.3) and solving for the derivative, results in the first EAF nonlinear differential equation,

$$
d r_{\text {eaf }} / d t=\left(k_{3} / k_{2}\right) r_{\text {eaf }}^{(-m f-3)} i_{\text {eaf }}^{2}-\left(k_{1} / k_{2}\right) r_{\text {eaf }}^{(n f-1)}
$$

Applying KVL to the EAF circuit yields the second EAF nonlinear differential equation,

$$
d i_{e a f} / d t=\left(1 / L_{e a f}\right)\left(v_{\mathrm{I}}-k_{3} r_{e a f}^{(-m f-2)} i_{e a f}\right)
$$

\section{B.3. Thyristor controlled reactor}


The TCR is represented by a back to back connection of a thyristor pair, in series with a RL circuit. The thyristor conduction is controlled with the firing angle $\alpha$ generating harmonic currents [45-46]. Figure B.3 shows the TCR model.

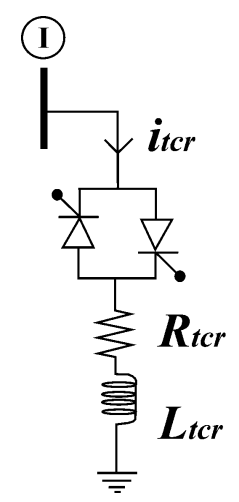

Figure B.3. Thyristor controlled reactor.

According to KVL,

$$
\begin{aligned}
& v_{\mathrm{I}}=v_{R t c r}+v_{L t c r}=i_{t c r} R_{t c r}+L_{t c r}\left(d i_{t c r} / d t\right) \\
& d i_{t c r} / d t=\left(v_{\mathrm{I}}-i_{t c r} R_{t c r}\right) / L_{t c r}
\end{aligned}
$$

The TCR current is dependent of the thyristor firing angle $\alpha$. Variable $s$ represents this dependency, defining the TCR nonlinear differential equation,

$$
d i_{t c r} / d t=s\left(v_{\mathrm{I}}-i_{t c r} R_{t c r}\right) / L_{t c r}
$$

\section{APPENDIX C}

\section{C.1. Definition of the state transition matrix $\boldsymbol{\Phi}$}

From (15),

$$
\boldsymbol{\Phi}_{k-1}=\partial \boldsymbol{f}_{k-1} / \partial \boldsymbol{x}=\partial\left[\boldsymbol{x}_{k-1}+\Delta t \boldsymbol{f}\left(\boldsymbol{x}_{k-1}, \boldsymbol{u}_{k-1}, \boldsymbol{v}_{k-1}\right)\right] / \partial \boldsymbol{x}
$$

As selected variable, in case of TCR, $\boldsymbol{x}(47)$ is the state variable for the TCR current, the state variable $\boldsymbol{x}(22)$ is the voltage of node 4 where the TCR is connected, the model is,

$$
d \boldsymbol{x}(47) / d t=\left(w_{0} s / L_{t c r}\right)\left(\boldsymbol{x}(22)-\boldsymbol{x}(47) R_{t c r}\right)
$$

Ф has only two elements different of zero in row 47 corresponding to the partial derivatives with respect of $\boldsymbol{x}(22)$ and $\boldsymbol{x}(47)$,

$$
\boldsymbol{\Phi}(47,22)=\partial\left[\boldsymbol{x}(47)+\Delta t\left(w_{0} s / L_{t c r}\right)\left(\boldsymbol{x}(22)-\boldsymbol{x}(47) R_{t c r}\right)\right] / \partial \boldsymbol{x}(22)
$$




$$
\begin{aligned}
& \boldsymbol{\Phi}(47,22)=\Delta t\left(w_{0} s / L_{t c r}\right) \\
& \boldsymbol{\Phi}(47,47)=\partial\left[\boldsymbol{x}(47)+\Delta t\left(w_{0} s / L_{t c r}\right)\left(\boldsymbol{x}(22)-\boldsymbol{x}(47) R_{t c r}\right)\right] / \partial \boldsymbol{x}(47) \\
& \boldsymbol{\Phi}(47,47)=1-\Delta t\left(w_{0} s R_{t c r} / L_{t c r}\right)
\end{aligned}
$$

$s$ is a function to control the thyristor switching, depending on the firing angle $\alpha$. $\boldsymbol{\Phi}$ is a partial derivative matrix of $n \times n$ order, $n$ states.

\section{C.2. Definition of the measurements matrix $\boldsymbol{H}$}

From (18),

$$
\boldsymbol{H}_{k}=\partial \boldsymbol{h}_{k-1} / \partial \boldsymbol{x}_{k}
$$

If the EAF real power $P_{3}$ is a measurement output variable, from (B.7),

$$
\boldsymbol{P}_{3}=\boldsymbol{v}_{\text {eaf }} \boldsymbol{i}_{\text {eaf }}=\left(k_{3} \boldsymbol{x}(46)^{(-m f-2)}\right) \boldsymbol{x}(45)^{2}
$$

$\boldsymbol{x}(45)$ is the EAF current and $\boldsymbol{x}(46)$ is the EAF arc radius, then,

$$
\begin{gathered}
\boldsymbol{H}(r n, 45)=\partial \boldsymbol{P}_{3} / \partial \boldsymbol{x}(45)=k_{3} \boldsymbol{x}(46)^{(-m f-2)} 2 \boldsymbol{x}(45) \\
\boldsymbol{H}(r n, 46)=\partial \boldsymbol{P}_{3} / \partial \boldsymbol{x}(46)=k_{3}\left((-m f-2) \boldsymbol{x}(46)^{(-m f-3)}\right)\left(\boldsymbol{x}(45)^{2}\right)
\end{gathered}
$$

Where $r n$ indicates the row number corresponding to the measurement $P_{3}$ (EAF real power) in the measurements equation (5), other values in this row are zero. $\boldsymbol{H}$ is $m \times n$ order, $m$ measurements and $n$ states. 
Table 1

State variables

\begin{tabular}{|c|c|}
\hline Description & State Variable \\
\hline Line current & $1-20$ \\
\hline Nodal voltage & $21-32$ \\
\hline Nodal load current & $33-43$ \\
\hline Nonlinear inductance flux & 44 \\
\hline EAF current and radius & $45-46$ \\
\hline TCR current & 47 \\
\hline
\end{tabular}

Table 2

Output measurement variables

\begin{tabular}{|c|c|}
\hline Description & Variable \\
\hline Line current & $1-20$ \\
\hline Nodal voltage & $21-32$ \\
\hline Nodal load current & $33-39$ \\
\hline EAF real power & $45-46$ \\
\hline
\end{tabular}

Table 3

Sampling frequency and execution time according to number of points per cycle (NPC)

\begin{tabular}{|c|c|c|c|}
\hline NPC & Points & $\begin{array}{c}\text { Sampling } \\
\text { frequency (Hz) }\end{array}$ & $\begin{array}{c}\text { Execution } \\
\text { time (s) }\end{array}$ \\
\hline 256 & 3072 & 15360 & 12.28 \\
\hline 512 & 6144 & 30720 & 27.92 \\
\hline 1024 & 12288 & 61440 & 64.81 \\
\hline 2048 & 24576 & 122880 & 130.14 \\
\hline
\end{tabular}

Table A.1

Electrical linear load p.u.

\begin{tabular}{|c|c|c|c|c|c|c|c|c|c|c|c|}
\hline Node & 2 & 3 & 4 & 5 & 6 & 9 & 10 & 11 & 12 & 13 & 14 \\
\hline Resistance & 36.88 & 21.2 & 41.8 & 26.32 & 17.86 & 67.8 & 22.2 & 57.14 & 32.78 & 29.64 & 26.84 \\
\hline Inductance & 1.57 & 1.05 & 5.13 & 12.5 & 2.66 & 12.04 & 3.45 & 11.1 & 12.5 & 3.45 & 4.0 \\
\hline
\end{tabular}

